\title{
Investigation of The Pain and Disability Situation of The Individuals Working "Home-Office" At Home At The Covid-19 Isolation Process
}

\author{
Nihan Ozunlu Pekyavaş ${ }^{1}$ and Engin Pekyavaş ${ }^{2}$ iD \\ ${ }^{1}$ Baskent University, Faculty of Health Sciences, Department of Physiotherapy and Rehabilitation, Turkey \\ ${ }^{2}$ Special Statistical Consultancy, Turkey \\ *Corresponding author: nozunlu@baskent.edu.tr
}

\begin{abstract}
The aim of our study is to examine pain and disability in low back, knee and shoulder regions of individuals working at home during COVID-19 isolation process. In our study, 87 volunteers were included in digital media by questionnaire with data collection method. Individuals who worked in the office before COVID-19 isolation but started working from home during the isolation were included in our study. In addition to the socio-demographic characteristics of the individuals, their situation about the working environment at home was questioned. In addition, pain in the low back, knee and shoulder regions was evaluated with an 11-point pain scale (EPPS). In order to determine the level of disability, individuals were asked to fill in the Oswestry Disability Index (for the lumbar region), Lysholm Knee Score (for the knee region) and the Disability of Arm, Shoulder and Hand Questionnaire (DASH) (for the shoulder region). It was seen that $86.2 \%$ preferred desk environment more frequently as home working environment and $65.5 \%$ spent 3 to 8 hours in this environment. During the home isolation process, the highest pain was observed for low back pain (50.6\%) in individuals (EPPS:4-5), then shoulder pain (44.8\%) (EPPS:5) and knee pain (35.6\%) (EPPS:4-5). When the levels of disability were evaluated, the Oswestry score was found 13.08 \pm 11.98 , the Lysholm score was found $91.20 \pm 12.23$ and the DASH score was found $9.61 \pm 16.78$. It has been observed that individuals working at home during COVID-19 isolation process may develop shoulder and knee pain, and mostly in the low back region, and all these pain are defined as moderate severity pain. However, when we look at the levels of disability, it was seen that low back pain, knee pain or shoulder pain did not pose a significant problem in the life of the patient and the level of disability was low.
\end{abstract}

Keywords

Pain, Low back, Shoulder, Knee, Covid-19 isolation process

\section{INTRODUCTION}

During the COVID-19 isolation process, many office workers started to continue their daily working life at home, as well as in the world. This work system, called "Home-Office", caused a decrease in physical activity in individuals as well as isolation. "Home-Office" work is a form of practice that is rapidly becoming widespread for both developed and developing countries, which enables individuals to work from home depending on the employer or to adapt their homes to economic activity through their own initiatives (Karakoyun, 2016).

Although this way of working is seen as a cost-effective way of working, protecting the health of the individual becomes an important factor. In addition to ergonomic arrangements such as adequate lighting and ventilation, an important factor for physiotherapists is to ensure that the individual's working ergonomics is maintained (Elison, 2011; Carayon \& Smith, 2000; Harrington $\&$ Walker, 2004). If the ergonomic arrangements provided in the workplace are not fully achieved in the working position, the individual may expect to 
develop pain and disability in various regions due to working in the same position for a long time. According to the studies, a positive relationship was found between various neck problems and work-related risk factors such as static neck and arm postures, sitting time and workplace design. Among other job characteristics, high quantitative job demands, having little effect on one's job status and limited rest breaks were found to be determinants of neck pain (Eriksen et al.,1999; Ariens et al., 2001; Shannon et al., 2001).

It should also be taken into consideration that working conditions at home may not be similar to offices, ergonomic working conditions can not be met, and therefore, pain may arise in individuals in various regions. For this reason, the purpose of our planned study is to examine the pain and disability in the low back, knee and shoulder regions of individuals working at home during the isolation process.

\section{MATERIALS AND METHODS}

The study protocol was approved by the University Ethics Committee and written consent was obtained from all participants of the study. In our study, 87 volunteers were included in the digital platform by questionnaire with data collection method. Individuals between the ages of 18-50 who worked at the office for at least 6-8 hours a day at the office before the COVID-19 isolation process, but started working from home during the isolation were included in our study. In addition to the socio-demographic characteristics of the individuals, their characteristics of home working environments (the environment they work in, the average duration of stay in this environment per day, the device used actively) were questioned.

Pain Assessment: Pain intensity in the waist, knee, and shoulder regions of the participants was evaluated with an 11-point pain scale (EPPS). Individuals were asked to make markings considering their pain at rest. In EPPS, which is a Likert type scale with values between 0-10, it was stated as "0 = I have no pain, $10=\mathrm{I}$ have unbearably pain". The results were interpreted by a specialist physiotherapist (Kwong \& Pathak, 2007).

Determining Disability Levels: In order to determine the level of disability, individuals were asked to fill in the Oswestry Disability Index (for the lumbar region), Lysholm Knee Scoring Scale (for the knee region) and Disabilities of the Arm, Shoulder and Hand Questionnaire (DASH) (for the shoulder region).

- Oswestry Disability Index: Oswestry Disability Index was calculated over 50 points. The options in each question were scored between 0-5. The total score was calculated by summing up the scores of the answers given to all questions.

According to the results;

$0 \%$ to $20 \%$ - Low back pain is not a major problem in the patient's life

$20 \%$ to $40 \%$ - Low back pain slightly restricts the patient's daily life

$40 \%$ to $60 \%$ - Low back pain severely restricts the patient's daily life

$60 \%$ to $80 \%$ low back pain, the patient's daily life is completely restricted

$80 \%$ to $100 \%$ - Bed-dependent patient (or symptoms are exaggerated)

The results were interpreted by a specialist physiotherapist (Fairbank et al., 1980; Fritz \& Irrgang, 2001).

- Lysholm Knee Score: The Lysholm Knee Score consists of 8 parameters, each scored differently. The total score is 100 . The sum of the scores of the answers marked by the individual was recorded as the score of the individual. The results were interpreted by a specialist physiotherapist (Collins et al, 2011).

- Disabilities of the Arm, Shoulder and Hand Questionnaire (DASH): The survey includes a 30item disability / symptom scale: function (21 items), symptom severity (six items) and psychological factors (three items); and two optional scales: business (four items) and sports / performing arts (four items). The individual marked the appropriate answer according to the 5point Likert system. Results were obtained between $0-100$. The results were interpreted by a specialist physiotherapist (Düger et al., 2006).

Statistical Analysis: According to the sample size analysis, a total of 87 individuals were needed for $80 \%$ power and 5\% type 1 error. The power analysis of our study was determined by considering the level of disability as the primary outcome. The data obtained from the participants were analyzed using the statistics program (SPSS) version 20.0 prepared for social sciences (IBM SPSS Statistics for Windows, Armonk, NY: IBM Corp.). The data were presented in the tables and graphs using percentages and mean as the central 
distribution criterion and standard deviation as the spread criterion. Confidence interval was accepted as $95 \%$.

\section{RESULTS}

The mean age of 87 individuals (59.8\% male, $40.2 \%$ female) participating in our study was $38.18 \pm 7.56$ years, and the average body mass index was $26.05 \pm 4.69 \mathrm{~kg} / \mathrm{m}^{2}$. It was seen that $86.2 \%$ preferred desk environment more frequently as home working environment and $65.5 \%$ spent 3 to 8 hours in this environment (Graph 1).

It was observed that there was no low back, shoulder or knee pain before the isolation process at home. During the home isolation process, the highest pain intensity was observed for back pain (50.6\%) (EPPS: 4-5) (Graph 2), then shoulder pain
(44.8\%) (EPPS: 5) (Graph 4) and knee pain (35.6\%) (EPPS: 4-5) (Graph 3) in participants. When the levels of disability were evaluated, the Oswestry score was found $13.08 \pm 11.98$, the Lysholm score was found $91.20 \pm 12.23$ and the DASH score was found $9.61 \pm 16.78$ in homeoffice working.

Considering the most seen pain symptoms of participants classified by working hours, it was seen that;

- Knee pain in 0-2 hours $(\% 63,6)$,

- Low back pain 3-5 hours $(\% 56,6)$,

- Shoulder pain 6-8 hours $(\% 66,6)$,

- Low back pain 9-11 hours (\%50),

- Low back pain 13-15 hours (\%50)

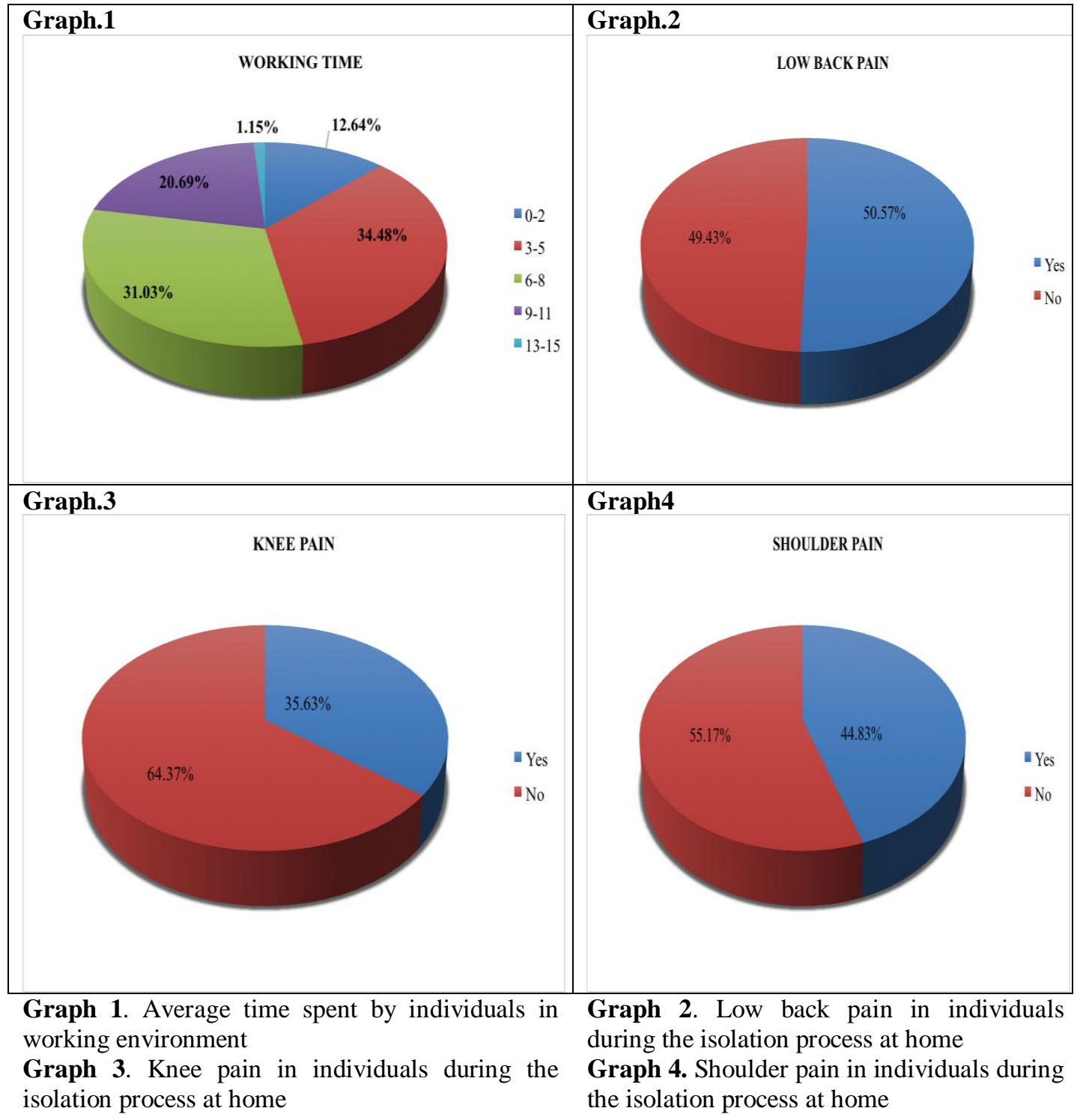




\section{DISCUSSION}

In this study, we aimed to examine the pain and disability conditions in the low back, knee and shoulder regions of individuals working at home during the COVID-19 isolation process and found that pain may occur in especially low back region more than knee and shoulder in individuals working at home, and all these pain may develop in moderate severity. Despite this, when we look at the levels of disability, it was seen that low back pain, knee pain or shoulder pain do not pose a significant problem in individuals' lives and their disability levels are found low.

Computer use in non-neutral postures such as neck rotation and shoulder abduction has been identified as risk factors for neck-shoulder symptoms (Tittiranonda, Burastero \& Rempel, 1999). Postural stress caused by poor workstation ergonomics, such as the inappropriate position of the screen, keyboard or mouse, has been associated with musculoskeletal problems (Demure et al., 2000). In our study, especially the low back, knee and shoulder regions that we think can be left under postural stress are chosen during working at home. With the working at home process, it was observed that individuals worked at the desk in the same position for high hours. However, it was observed that there was moderate pain revealed in each region evaluated due to postural stress. However, it has been observed that disability levels of individuals are not affected due to this pain. This is thought to be due to the fact that the posture created at home, together with the release of pain, may not have caused serious injury.

Low back region is though to be one of the most negatively affected areas in long term sitting during work hours. Although this situation, the extensive recent epidemiological literature does not support the popular opinion that sitting-whileat-work is associated with low back pain (Hartvigsen et al., 2000; Roffey et al., 2010). Neck, back, shoulder and knee/thigh areas are also found to be the highest discomfort prevalence rates ranging from $35 \%$ to $60 \%$, and about $90 \%$ of the discomfort was related to bus-driving in bus drivers who are also working in prolonged sitting position (Szeto \& Lam, 2007). In our study, we have seen that pain may occur in especially low back region more than knee and shoulder in individuals working at home, and all these pains may develop in moderate severity which did not pose a significant problem in individuals' lives and their disability levels are found low.

According to a study, the influence of the home office is found to appear to be mostly positive and the influence of traditional office mostly negative on aspects of both work and personal/life which includes work/life balance and personal/family success (Hill, Ferris \& Martinson, 2003). Another possible reason for our study results might depend on this situation, which would affect the pain perception in participants. Working from home might have caused the participants to have less pain although there are improper working conditions as we all are biopsychosocial beings.

We thought that the individuals who were working in the office before the COVID-19 isolation process and started to work at home with the isolation process, would have developed pain and disability more in the low back, knee and shoulder regions due to the lack of ergonomic working order and a decrease in long-term physical activity. However, the results of our study show us that it does not pose a significant problem in individuals' lives. Considering the limitations of our study, we think that the inclusion of other regions, such as the neck, which may be affected by the posture, can provide different information about the level of pain and disability of individuals during the work at home. Also identifying the previous physical activity levels and working postures may be more informative about the subjects.

Conflict of interest: The authors declare no conflict of interest. No financial support was received.

\section{REFERENCES}

Ariens, G., Bongers, P., Hoogendoorn, W., Houtman, I.L.D., van der Wal, G., van Mechelen, W. (2001). High quantitative job demands and low coworker support as risk factors for neck pain: results of a prospective cohort study. Spine, 26:1896-901.

Carayon, P., Smith, M. J. (2000). Work organization and ergonomics. Appl Ergon, 31(6), 649-662.

Collins, N.J., Misra, D., Felson, D.T., Crossley, K.M., Roos, E. M. (2011). Measures of knee function: International Knee Documentation 
Committee (IKDC) Subjective Knee Evaluation Form, Knee Injury and Osteoarthritis Outcome Score (KOOS), Knee Injury and Osteoarthritis Outcome Score Physical Function Short Form (KOOS-PS), Knee Outcome Survey Activities of Daily Living Scale (KOS-ADL), Lysholm Knee Scoring Scale, Oxford Knee Score (OKS), Western Ontario and McMaster Universities Osteoarthritis Index (WOMAC), Activity Rating Scale (ARS), and Tegner Activity Score (TAS). Arthritis Care Res, 63(S11), 208-228.

Demure, B., Luippold, R., Bigelow, C., Danielle, A., Mundt, K.A., Bernhard, L. Video display terminal workstation improvement program: I. Baseline associations between musculoskeletal discomfort and ergonomic features of workstations. J Occup Environ Med 2000;42:783-91.

Düger, T., Yakut, E., Öksüz, Ç., Yörükan, S., Bilgütay, B. S., Ayhan, Ç., Leblebicioğlu, G., Kayıhan, H., Kırdı, N., Yakut, Y., Güler, Ç. (2006). Reliability and validity of the Turkish version of the Disabilities of the Arm, Shoulder and Hand (DASH) Questionnaire. Physiotherapy Rehabilitation, 17(3), 99-107.

Ellison, J. K. (2011). Ergonomics for telecommuters and other remote workers. In ASSE Professional Development Conference and Exposition. American Society of Safety Engineers.

Eriksen, W., Natvig, B., Knardahl, S., Bruusgaard, D. (1999). Job characteristics as predictors of neck pain. A 4-year prospective study. $J$ Occup Environ Med, 41:893-902.

Fairbank, J.C., Couper, J., Davies, J.B., O'Brien, J.P. (1980). The Oswestry low back pain disability questionnaire. Physiotherapy, 66, 271-273.

Fritz, J.M., Irrgang, J.J. (2001). A comparison of a modified Oswestry Low Back Pain Disability Questionnaire and the Quebec Back Pain Disability Scale. Phys Ther, 81, 776-788.

Harrington, S. S., Walker, B. L. (2004). The effects of ergonomics training on the knowledge, attitudes, and practices of teleworkers. J Safety Res, 35(1), 13-22.
Hartvigsen, J., Leboeuf-Yde, C., Lings, S., \& Corder, E. H. (2000). Review Article: Is sitting-while-at-work associated with low back pain? A systematic, critical literature review. Scandinavian Journal of Public Health, 28(3),230-239. doi.10.1177/14034948000280030201

Hill, E. J., Ferris, M., \& Märtinson, V. (2003). Does it matter where you work? A comparison of how three work venues (traditional office, virtual office, and home office) influence aspects of work and personal/family life. Journal of Vocational Behavior, 63(2), 220-241.

Karakoyun, F. (2016). Home Office -Working at Home- System and Adjustment on Taxation Law. Int. Journal of Management Economics and Business, 12(29), 141-162.

Kwong, W.J., Pathak, D.S. (2007). Validation of the eleven-point pain scale in the measurement of migraine headache pain. Cephalalgia, 27(4), 336-342.

Roffey, D. M., Wai, E. K., Bishop, P., Kwon, B. K., \& Dagenais, S. (2010). Causal assessment of occupational sitting and low back pain: results of a systematic review. The Spine Journal, 10(3), 252-261.

Shannon, H., Woodward, C., Cunningham, C., McIntosh, J., Lendrum, B., Brown, J., \& Rosenbloom, D. (2001). Changes in general health and musculoskeletal outcomes in the workforce of a hospital undergoing rapid change: a longitudinal study. J Occup Health Psychol, 2001;6:3-14.

Szeto, G. P., \& Lam, P. (2007). Work-related musculoskeletal disorders in urban bus drivers of Hong Kong. Journal of occupational rehabilitation, 17(2), 181-198.

Tittiranonda, P., Burastero, S., Rempel, D. (1999). Risk factors for musculoskeletal disorders among computer users. Occupational Medicine: State of the Art Reviews,14:17-38.

How to cite this article: Pekyavaş, N.Ö. and Pekyavaş, E. (2020). Investigation of The Pain and Disability Situation of The Individuals Working "Home-Office" At Home At The Covid-19 Isolation Process. Int J Disabil Sports Health Sci;3(2):100-104. https://doi.org/10.33438/ijdshs. 772675 